\title{
Alterações no estado nutricional segundo IMC e perda de peso, em pacientes com câncer de cabeça e pescoço em uso de terapia nutricional enteral, em ambulatório de oncologia clínica em São Paulo
}

\author{
Changes in nutritional status according to BMI and weight loss in patients with head and neck cancer \\ using enteral nutritional therapy in a clinical oncology outpatient clinic in São Paulo
}

DOI: $10.37111 /$ braspenj.2020351005

Nathalia Soares Machado

Julia de Castro Querido²

Mayara Freitas de Oliveira ${ }^{3}$

Lidiane Pereira Magalhães ${ }^{4}$

\section{Unitermos:}

Neoplasias de cabeça e pescoço. Avaliação nutricional. Nutrição enteral. Estado nutricional.

\section{Keywords:}

Head and neck neoplasms. Nutrition assessment. Enteral nutrition. Nutritional status.

\section{Endereço para correspondência:}

Nathalia Soares Machado

Universidade Federal de São Paulo - UNIFESP - campus São Paulo

Departamento de Oncologia Clínica e Experimental Rua Doutor Diogo de Faria, 824 - Vila Clementino São Paulo, SP, Brasil - CEP 04037-002

E-mail: nathaliamachado.nutri@gmail.com

\section{Submissão}

16 de outubro de 2019

Aceito para publicação

10 de março de 2020
RESUMO

Introdução: Pacientes com câncer de cabeça e pescoço (CCP) configuram um reconhecido grupo de risco para desnutrição devido a diversos fatores, podendo necessitar de terapia nutricional enteral (TNE). Este trabalho avaliou alterações no estado nutricional e a perda de peso de pacientes com CCP em uso de TNE, acompanhados no ambulatório de oncologia clínica da Universidade Federal de São Paulo (UNIFESP). Método: Estudo retrospectivo realizado por meio da análise de banco de dados, coletados no período de janeiro a dezembro de 2018, em ambulatório de oncologia clínica da UNIFESP. Foram selecionados todos os pacientes com CCP que utilizaram TNE em algum momento do tratamento, durante o ano de 2018. Consideraram-se as variáveis peso e altura, para o cálculo do índice de massa corporal (IMC) e classificação do estado nutricional e a classificação da perda de peso para avaliação. Resultados: A amostra foi composta por 24 pacientes, sendo $71 \%$ de idosos e $29 \%$ adultos e 75\% eram homens. Na primeira consulta de 2018, 6 (25\%) pacientes eram eutróficos e 15 (63\%) apresentaram algum grau de desnutrição (baixo peso). Um total de 11 (46\%) pacientes referiu perda de peso prévia. Após a análise, verificou-se que $54 \%$ dos pacientes apresentaram perda ponderal no período. Destes, apenas 1 paciente apresentou perda ponderal grave. Foram observadas alterações no IMC em todos os pacientes, porém em $88 \%$ dos casos não houve alteração na classificação do IMC. Conclusão: Apesar dos pacientes estarem em TNE, muitos apresentaram perda de peso, o que pode ser resultado da piora na ingestão alimentar, de anormalidades metabólicas causadas pela atividade tumoral, ou consequência da sintomatologia relacionada ao tratamento. As perdas ponderais foram, em sua maioria, não significativas, e boa parte dos pacientes teve aumento do peso corporal, o que pode ter sido resultado da TNE, que é responsável pelo retardo na perda de peso em pacientes oncológicos.

\section{ABSTRACT}

Introduction: Head and neck cancer (HNC) patients constitute a recognized risk group for malnutrition due to several factors, and may require enteral nutritional therapy (ENT). This study evaluated alterations in nutritional status and weight loss in patients with HNC using ENT followed at the Universidade Federal de São Paulo (UNIFESP) oncology outpatient clinic. Methods: This was a retrospective study conducted through database analysis, collected from January to December of 2018 at an UNIFESP oncology outpatient clinic. We selected all patients with HNC in use of ENT at some moment of the treatment during 2018. Weight and height were considered to calculate body mass index (BMI) and to classify nutritional status and the classification of weight loss for evaluation. Results: The sample consisted of 24 patients, $71 \%$ were elderly and $29 \%$ adults and $75 \%$ were men. At the first consultation in 2018,6 $(25 \%)$ patients were at normal weight and $15(63 \%)$ had some degree of malnutrition (underweight). A total of $11(46 \%)$ patients reported previous weight loss. After the analysis it was found that $54 \%$ of patients had weight loss in the period, however, only 1 patient had severe weight loss. Changes in $\mathrm{BMl}$ were observed in all patients, but in $88 \%$ of the cases there was no change in BMI classification. Conclusion: Although patients were on ENT, many had weight loss, which may be a result of decreased food intake, metabolic abnormalities caused by tumor activity, or a consequence of treatment-related symptoms. Most of the weight losses were not significant, and most patients had increased body weight, which may have been result of ENT use, which is responsible for delayed weight loss in cancer patients.

1. Residente do Programa de Residência Multiprofissional em Oncologia da Universidade Federal de São Paulo (UNIFESP); Especialista em Nutrição Esportiva pelo Centro de Estudos de Fisiologia do Exercício e Treinamento (CEFIT); Graduação em Nutrição pela Universidade de São Paulo (USP), onde foi bolsista da Coordenação de Aperfeiçoamento de Pessoal de Nível Superior (CAPES), com período sanduíche na La Salle University (2015/2016), São Paulo, SP, Brasil.

2. Residente do Programa de Residência Multiprofissional em Oncologia da Universidade Federal de São Paulo (UNIFESP); Graduação em Nutrição na Universidade Federal de Alfenas (UNIFAL), onde foi bolsista da Coordenação de Aperfeiçoamento de Pessoal de Nível Superior (CAPES), com período sanduíche na Northumbria University (2014/2015), São Paulo, SP, Brasil.

3. Residente do Programa de Residência Multiprofissional em Oncologia da Universidade Federal de São Paulo (UNIFESP); Graduação em Nutrição pela Universidade de São Paulo (USP), São Paulo, SP, Brasil.

4. Doutoranda em Ciências da Saúde na área Oncologia na Universidade Federal de São Paulo (UNIFESP); Mestrado em Ciências da Saúde na área de Oncologia na UNIFESP; Especialista em Nutrição Clínica pelo GANEP; Graduação em Nutrição Universidade São Judas Tadeu, São Paulo, SP, Brasil. 


\section{INTRODUÇÃO}

câncer de cabeça e pescoço (CCP) compreende um conjunto de neoplasias que podem se manifestar na boca, faringe, laringe, entre outras regiões da cabeça e do pescoço'. Segundo estimativas do Instituto Nacional do Câncer (INCA), é atualmente o segundo tipo de câncer mais frequente entre os homens no Brasil, atrás somente do câncer de próstata².

Pacientes com CCP configuram um reconhecido grupo de risco para desnutrição e caquexia ${ }^{3,4}$. A perda de peso associada ao câncer é resultado tanto da redução na ingestão alimentar quanto das alterações metabólicas provocadas pelo tumor e, apesar da diminuição na ingestão alimentar ser comum em pacientes com todos os tipos de tumores sólidos, nos pacientes com câncer de cabeça e pescoço, a piora no padrão alimentar é mais significativa, devido aos sintomas e efeitos colaterais do tratamento ${ }^{3}$.

Esse grupo de pacientes comumente apresenta sintomas relacionados à localização tumoral, como trismo, disfagia, odinofagia, dificuldade de deglutição por obstrução mecânica e dores na cavidade oral ${ }^{4-6}$. Além desses sintomas relacionados à localização do tumor, efeitos sistêmicos do câncer podem resultar em alterações no paladar e no apetite, dificultando ainda mais a adequação alimentar desses pacientes. A junção dessas alterações e limitações pode levar à necessidade do uso de vias alternativas de alimentação ${ }^{5-7}$.

Os principais tratamentos para esse tipo de câncer são cirurgia, radioterapia, quimioterapia ou a combinação destes que também resultam em efeitos colaterais, como náusea, vômitos, mucosite, xerostomia, alteração ou perda do paladar, odinofagia e perda de apetite, podendo contribuir ainda mais para perda ponderal e desnutrição $0^{5,7}$.

Por sua vez, o quadro de desnutrição está associado a maiores taxas de mortalidade e morbidade, com maior risco de infecções, declínio da qualidade de vida e status de funcionalidade, piora de sobrevida, além de adicionar custos aos cuidados dos pacientes ${ }^{4-6}$.

Em estudos com esse perfil de pacientes, a desnutrição é geralmente definida como perda de peso não intencional maior que $5-10 \%$ durante os últimos 1 a 6 meses e IMC menor que 18,5 a $20^{4}$. Pacientes com perda de peso apresentam morbidades relacionadas ao tratamento mais graves e de maior duração, além de apresentarem mais complicações clínicas, como diminuição da capacidade de cicatrização de feridas, redução da função imunológica e menor tolerância a procedimentos cirúrgicos, quimioterapia e radioterapia ${ }^{4,5}$.

Considerando sintomas relacionados ao tumor, e também ao tratamento antineoplásico, se uma intervenção nutricional não for iniciada, a perda de peso durante o tratamento irá se perpetuar ${ }^{5}$.
A intervenção nutricional e a suplementação têm papel importante na prevenção da deterioração do estado nutricional e ingestão alimentar. $\bigcirc$ suporte nutricional tem o objetivo de prevenir o hipercatabolismo e a redução da ingestão alimentar associados à caquexia e pode diminuir o processo catabólico em pacientes que já se encontram com caquexia ${ }^{4}$.

A manutenção ou melhora do estado nutricional, realizada por meio do suporte nutricional precoce, pode permitir que $\circ$ paciente conclua o tratamento planejado, evitando interrupções, reduzindo sua toxicidade e conferindo melhor prognóstico ao paciente ${ }^{4,8}$.

Tendo em vista a gravidade das consequências nutricionais desse tipo de tumor e tratamentos relacionados, e a importância da terapia nutricional, o objetivo deste estudo é avaliar as alterações no estado nutricional e a perda de peso de pacientes com diagnóstico de CCP em uso de terapia nutricional enteral acompanhados no ambulatório de oncologia clínica da Universidade Federal de São Paulo (UNIFESP).

\section{MÉTODO}

Esse estudo retrospectivo foi realizado por meio da análise de um banco de dados no Microsoft Excel $\AA$ pertencente ao departamento de Nutrição em Oncologia, inserido no Departamento de Oncologia Clínica e Experimental da Universidade Federal de São Paulo (UNIFESP). Os dados utilizados no estudo foram coletados durante as consultas, no período de janeiro a dezembro de 2018, em um ambulatório de Oncologia Clínica da UNIFESP.

\section{Participantes do Estudo e Critérios de Exclusão}

Inicialmente, foram selecionados todos os pacientes com idade superior a 18 anos, atendidos em um ambulatório de oncologia clínica da UNIFESP, com diagnóstico de CCP, do tipo histológico carcinoma espinocelular, incluindo diversos sítios, que utilizaram terapia nutricional enteral em algum momento do tratamento oncológico, durante o ano de 2018. Não participaram da amostra pacientes que compareceram em apenas uma consulta no período de janeiro a dezembro de 2018, devido à impossibilidade de comparação intrapessoal para avaliação de alterações do estado nutricional. A amostra foi estratificada por idade.

\section{Variáveis Antropométricas}

Uma das variáveis analisadas para determinar o estado nutricional dos pacientes foi o índice de massa corporal (IMC), calculado após a aferição do peso e altura dos pacientes, considerando variações mínimas de 0,1 kg e 0,1 $\mathrm{cm}$, respectivamente. $\mathrm{O}$ cálculo do IMC foi realizado pela 
fórmula [(peso em kg)/(altura em m) ${ }^{2}$. Para classificar o IMC dos pacientes, foi utilizada a referência da Organização Mundial da Saúde $(\mathrm{OMS})^{9}$ para adultos, classificando o estado nutricional em baixo peso quando $<18,5 \mathrm{~kg} / \mathrm{m}^{2}$, eutrofia $\geq 18,5 \mathrm{~kg} / \mathrm{m}^{2}$ e $<25,0 \mathrm{~kg} / \mathrm{m}^{2}$, sobrepeso quando $\geq 25,0 \mathrm{~kg} / \mathrm{m}^{2}$ e $<30,0 \mathrm{~kg} / \mathrm{m}^{2}$ e obesidade $\geq 30,0 \mathrm{~kg} / \mathrm{m}^{2}$, e para idosos, foi utilizada a referência da Organização Pan Americana da Saúde $(\mathrm{OPAS})^{10}$, classificando o estado nutricional em baixo peso quando $\leq 23,0 \mathrm{~kg} / \mathrm{m}^{2}$, eutrofia maior que 23,0 e menor que $28,0 \mathrm{~kg} / \mathrm{m}^{2}$, sobrepeso quando $\geq 28,0$ e $<30,0 \mathrm{~kg} / \mathrm{m}^{2}$ e obesidade se $\geq 30,0 \mathrm{~kg} / \mathrm{m}^{2}$.

Foi realizada comparação individual entre o IMC dos participantes na primeira e na última consulta de 2018. A alteração intrapessoal do IMC foi constatada e dividida em quatro classificações: diminuição ou aumento significativo do IMC, quando houve alteração na classificação do mesmo, e diminuição ou aumento não significativo quando apesar de ter havido alteração no valor, o IMC se manteve na mesma classificação.

Além do IMC, foi observada perda de peso prévia à primeira consulta no ambulatório de Oncologia Clínica, e a perda de peso dos pacientes entre a primeira e a última consulta no ambulatório no ano de 2018. O percentual de perda de peso entre as consultas, calculado pela fórmula [(peso inicial - peso atual) x 100/peso inicial], foi classificado de acordo com o critério de Blackburn et al. ${ }^{11}$, o que resultou nas classificações "significativa" e "grave", conforme pode ser observado na Tabela 1. Os valores que estavam fora do intervalo da referência foram classificados como perda de peso "não significativa".

Tabela 1 - Classificação da porcentagem de perda de peso de acordo com o tempo.

\begin{tabular}{lcc}
\hline \multirow{2}{*}{ Tempo } & \multicolumn{2}{c}{ Classificação } \\
\cline { 2 - 3 } & Significativa (\%) & Grave (\%) \\
\hline 1 semana & $1-2$ & $>2$ \\
1 mês & 5 & $>5$ \\
3 meses & 7,5 & $>7,5$ \\
6 meses & 10 & $>10$ \\
\hline
\end{tabular}

Fonte: Blackburn et al. ${ }^{11}$

\section{Análise e Apresentação de Resultados}

Para a tabulação dos dados e cálculos foi utilizado o Microsoft Excel ${ }^{\circledR}$. Os dados analisados foram apresentados em valores absolutos e porcentagem. Variáveis como idade e IMC foram apresentadas também em média e desvio padrão.

\section{RESULTADOS}

\section{Participantes do Estudo}

A amostra foi composta por 24 pacientes, sendo $71 \%$ $(n=17)$ idosos e $29 \%$ adultos $(n=7)$ e $75 \%$ dos pacientes eram homens $(n=18)$. A média de idade dos adultos foi de $54 \pm 3,4$ anos e a média de idade dos idosos foi de $67 \pm$ 4,5 anos.

Dos 24 participantes, 18 (75\%) apresentavam comorbidades, dentre elas: hipertensão arterial sistêmica, diabetes mellitus, dislipidemias, cardiopatias, hipotireoidismo, doença pulmonar obstrutiva crônica (DPOC) e depressão.

Entre os diferentes sítios de localização primária do tumor estavam: faringe, laringe e cavidade oral.

Os dados referentes ao perfil da população do estudo são apresentados na Tabela 2.

\begin{tabular}{|c|c|c|}
\hline $\begin{array}{l}\text { Idade } \\
\text { (anos, média } \pm \text { DP) }\end{array}$ & $\begin{array}{l}\text { Adulto } \\
54 \pm 3,4\end{array}$ & $\begin{array}{l}\text { Idoso } \\
67 \pm 4,5\end{array}$ \\
\hline & Significativa (\%) & Grave (\%) \\
\hline \multicolumn{3}{|l|}{ Sexo } \\
\hline Masculino & 75 & 18 \\
\hline Feminino & 25 & 6 \\
\hline \multicolumn{3}{|l|}{ Faixa etária } \\
\hline Idoso (>60 anos) & 71 & 17 \\
\hline Adulto (18 a 59 anos) & 29 & 7 \\
\hline \multicolumn{3}{|l|}{ Comorbidades } \\
\hline $\begin{array}{l}\text { Hipertensão arterial } \\
\text { sistêmica }\end{array}$ & 41,7 & 10 \\
\hline Diabetes & 8,3 & 2 \\
\hline Dislipidemias & 4,2 & 1 \\
\hline Cardiopatias & 8,3 & 2 \\
\hline Hipotireoidismo & 16,7 & 4 \\
\hline $\begin{array}{l}\text { Doença pulmonar } \\
\text { obstrutiva crônica }\end{array}$ & 12,5 & 3 \\
\hline Depressão & 8,3 & 2 \\
\hline \multicolumn{3}{|l|}{ Localização do tumor } \\
\hline Faringe & 25,0 & 6 \\
\hline Laringe & 41,7 & 10 \\
\hline Cavidade oral & 33,3 & 8 \\
\hline
\end{tabular}

\section{Estado Nutricional}

Na primeira consulta de 2018, 6 (25\%) pacientes eram eutróficos, 15 (63\%) apresentaram algum grau de desnutrição (baixo peso) e 3 (12\%) apresentaram excesso de peso (obesidade ou sobrepeso). A média do IMC na primeira consulta entre os adultos foi de $20,9 \pm 5,6 \mathrm{~kg} / \mathrm{m}^{2}$ e entre os idosos foi de $20,8 \pm 4,1 \mathrm{~kg} / \mathrm{m}^{2}$.

Na última consulta de 2018, 5 (21\%) pacientes eram eutróficos, 17 (71\%) apresentaram algum grau de desnutrição (baixo peso) e 2 (8\%) apresentaram excesso de peso (sobrepeso ou obesidade). A média do IMC na última consulta entre os adultos foi de $21,3 \pm 5,5 \mathrm{~kg} / \mathrm{m}^{2}$ e entre os idosos foi de $20,5 \pm 4,4 \mathrm{~kg} / \mathrm{m}^{2}$. 
Analisando as alterações intrapessoais do IMC dos pacientes entre a primeira e a última consulta de 2018, foram observadas alterações no IMC em todos os pacientes, sendo que em $88 \%$ dos casos não houve alteração na classificação do IMC. Ao detalhar esses dados, foi possível identificar que 13 pacientes apresentaram diminuição do IMC, sendo que 3 (12\%) apresentaram diminuição significativa e 10 (42\%), diminuição não significativa, segundo critério previamente estabelecido. Nenhum paciente apresentou aumento significativo no IMC, e 11 (46\%) doentes exibiram aumento não significativo.

Ao observar o critério perda de peso, um total de 11 (46\%) pacientes referiu perda de peso prévia à primeira consulta.

Após a análise dos dados, verificou-se que $54 \%$ dos pacientes $(n=13)$ apresentaram perda ponderal no período entre consultas. Destes, apenas 1 (8\%) paciente apresentou perda ponderal grave, de acordo com a classificação por percentual de perda de peso, e 12 (92\%) tiveram perda não significativa.

\section{DISCUSSÃO}

Ao observar o perfil dos pacientes do estudo, é possível verificar que a maioria é constituída por indivíduos do sexo masculino e idosos, o que está de acordo com achados de estudos prévios com pacientes portadores de neoplasia de cabeça e pescoço ${ }^{3,12-14}$. A predominância de indivíduos do sexo masculino pode estar relacionada à maior exposição aos principais fatores de risco, como o tabaco, cuja prevalência de consumo no Brasil é maior em homens do que em mulheres, e fatores de proteção, visto que consultas odontológicas com regularidade costumam ser mais frequentes entre mulheres, e as diferenças socioeconômicas entre gêneros ${ }^{13}$.

Segundo a Organização Mundial da Saúde, citada por Theme Filha et al. ${ }^{15}$, são consideradas doenças crônicas não transmissíveis (DCNT) as doenças cerebrovasculares, cardiovasculares, diabetes mellitus, doenças respiratórias obstrutivas, asma e neoplasias. Também se inclui no rol de condições crônicas os transtornos psiquiátricos como depressão. Em estudo de Theme Filha et al. ${ }^{15}$, que avaliou a prevalência de DCNT, foi verificado que as mais prevalentes foram hipertensão arterial $(21,4 \%)$, seguido por depressão $(7,6 \%)$ e diabetes mellitus $(6,2 \%)$. Nos indivíduos que participaram deste estudo, observou-se maior prevalência de hipertensão arterial $(41,7 \%)$, seguido de hipotireoidismo $(16,7 \%)$, doença pulmonar obstrutiva crônica (12,5\%), e cardiopatias, depressão e diabetes mellitus, as três encontradas em 8,3\% dos pacientes. Comparando com os achados de Theme Filha et al. ${ }^{15}$, pode-se notar uma semelhança, sendo a DCNT mais prevalente a hipertensão arterial em ambos os estudos, e também muito frequentes aparecem a depressão e o diabetes mellitus. No presente estudo, a prevalência de doença pulmonar obstrutiva foi bastante importante, o que pode estar relacionado com o perfil dos indivíduos do estudo, em sua maioria usuários ou ex-usuários de tabaco, que é definido como um fator de risco para o desenvolvimento de ambas as doenças DPOC e CCP 16,17 .

Ao analisar os critérios perda de peso e estado nutricional baseado no IMC, foi possível observar que $46 \%$ dos pacientes referiram perda de peso prévia à primeira consulta e $63 \%$ apresentavam algum grau de desnutrição (baixo peso). $\mathrm{Na}$ literatura, a perda de peso grave e desnutrição prévia ao início do tratamento é amplamente relatada e estima-se que cerca de $19 \%$ a $57 \%$ dos pacientes com câncer de cabeça e pescoço sejam desnutridos ao diagnóstico ${ }^{4,12}$. Em um estudo de Mulasi et al. ${ }^{12}$, identificou-se prevalência de $67 \%$ de desnutrição ao diagnóstico, o que está de acordo e próximo à prevalência observada no atual estudo. $\mathrm{Na}$ fase pré-tratamento, esse quadro se deve principalmente ao câncer em $\mathrm{si}^{4}$. Os sintomas relacionados ao tumor e à localização do mesmo, que incluem obstrução mecânica, dificuldade de deglutição, disfagia, odinofagia, trismo e dores na cavidade oral, podem dificultar muito o processo de alimentação, além da associação a efeitos sistêmicos do câncer que podem resultar em alteração de paladar e apetite, prejudicando ainda mais a ingestão alimentar ${ }^{4-6}$. A anorexia induzida pelo tumor é resultado da alteração de neurotransmissores, hormônios imunorreguladores e aversão adquirida a certos alimentos ${ }^{17}$. Além disso, a perda de peso pode resultar de alterações metabólicas relacionadas ao tumor, como o aumento no gasto energético, proteólise e lipólise ocasionadas pela presença de inflamação sistêmica ${ }^{18}$.

Um fator externo que também pode corroborar com esse achado, é que pacientes com CCP tem, usualmente, risco de desnutrição crônica devido a maus hábitos alimentares em decorrência do excessivo consumo de álcool e tabaco, que comprometem a adequação da ingestão alimentar ${ }^{5,17}$. O uso de tabaco e álcool, muito comum nesse grupo de pacientes, fornece aos usuários calorias vazias, desprovidas de nutrientes essenciais e causa supressão do apetite, agravando ainda mais o quadro de desnutrição ${ }^{17}$.

Após comparação do estado nutricional, segundo as variáveis analisadas entre a primeira e a última consulta, foi possível identificar que todos os pacientes tiveram alteração no IMC, sendo que em $88 \%$ dos casos não houve alteração na classificação do mesmo. Além disso, a prevalência de indivíduos com algum grau de desnutrição (baixo peso) de acordo com o IMC passou de 63\%, na primeira consulta, para $71 \%$, na última. Um total de 13 (54\%) pacientes apresentaram perda de peso e, consequentemente, diminuição do IMC e, em 8\% dos casos, essa perda ponderal foi classificada como grave para o período. No estudo de Mulasi et al. ${ }^{12}$, já citado anteriormente, foi observado que o número de pacientes desnutridos aumentou durante e após 
a concretização do tratamento e que após o tratamento $82 \%$ dos pacientes continuaram apresentando perda de peso. É relatado na literatura que, durante a radioterapia isoladamente ou em concomitância com quimioterapia, a desnutrição se apresenta em $44 \%$ a $88 \%$ dos pacientes ${ }^{4}$. Considerando o encontrado no estudo, onde a maioria dos pacientes apresentou perda de peso e diminuição do IMC entre as consultas, é possível relacionar esse fato às consequências nutricionais ocasionadas pelo tratamento em associação às alterações já citadas ocasionadas pelo tumor. Ao discorrer sobre a perda de peso ocasionada pelas consequências do tratamento oncológico, é plausível citar efeitos colaterais da quimioterapia e da radioterapia relacionados ao trato gastrointestinal, como náuseas, vômito, disfagia, odinofagia, xerostomia, mucosite, alterações no paladar e perda de apetite ${ }^{4,5}$. Além disso, intervenções cirúrgicas, dependendo de sua extensão, podem alterar as etapas da deglutição e digestão, implicando, assim, em desequilíbrio nutricional desses pacientes ${ }^{14,19}$.

Um estudo de Camargo et al. ${ }^{20}$ avaliou a variação do IMC de pacientes portadores de neoplasias de cabeça e pescoço, em uso de sonda nasogástrica (SNG), e constatou melhora ou manutenção do IMC com a inserção da SNG durante o período avaliado. Nosso estudo constatou que 11 (46\%) pacientes apresentaram aumento do IMC no período observado e que, apesar de $54 \%$ dos pacientes terem apresentado perda ponderal e diminuição do IMC, em $92 \%$ dos casos as perdas ponderais foram classificadas como não significativas, segundo critério previamente estabelecido, e para $88 \%$ dos pacientes não houve alteração na classificação do IMC. Esse fato pode ser justificado pelo impacto positivo da terapia nutricional enteral (TNE) na composição corporal dos pacientes. Estudos demonstram que esta desempenha papel importante no retardo da perda de peso, melhora na ingestão alimentar, prevenção da deterioração do estado nutricional, garantindo o desempenho de sistemas vitais, como a capacidade de cicatrização e função imunológica, e na recuperação do estado funcional, o que afeta positivamente a qualidade de vida dos pacientes 4,8,19,20.

Além do impacto da TNE, o aconselhamento nutricional de indivíduos durante o tratamento de quimioterapia e radioterapia demonstrou efeitos positivos na ingestão alimentar e nos parâmetros nutricionais dos pacientes ${ }^{4}$. Além disso, a melhora do estado nutricional está relacionada à redução da toxicidade da terapia antineoplásica, permitindo que o paciente conclua o tratamento planejado com reduzida morbidade $4,8,20$.

\section{CONCLUSÃO}

Apesar dos pacientes estarem em terapia nutricional, muitos ainda apresentaram perda de peso, o que pode ser resultado da piora na ingestão alimentar ocasionada pela localização do tumor, de anormalidades metabólicas causadas pela atividade tumoral, ou consequência da sintomatologia relacionada ao tratamento. As perdas ponderais foram, em sua maioria, não significativas, e muitos pacientes tiveram aumento do peso corporal, o que pode ter sido resultado da terapia nutricional, visto que é observado que a mesma é responsável pelo retardo na perda de peso em pacientes oncológicos.

Dessa forma, fica evidente que a intervenção nutricional é determinante para a redução dos efeitos colaterais e continuidade do tratamento, para a melhora do perfil nutricional, e auxílio na qualidade de vida dos pacientes.

\section{REFERÊNCIAS}

1. Castro G. Câncer de cabeça e pescoço é um dos tumores mais frequentes em homens [Internet]. Brasil: Hospital Sírio-Libanês; 21 de julho de 2017 [citado em 28 de junho de 2019]. Disponível em: https://www.hospitalsiriolibanes.org.br/sua-saude/Paginas/ cancer-cabeca-pescoco-tumores-mais-frequentes-homens.aspx

2. Instituto Nacional do Câncer. Prevenção e detecção precoce do câncer de cabeça e pescoço são tema de encontro no INCA [Internet]. Rio de Janeiro; 28 de julho de 2016 [atualizado em 08 de março de 2018; citado em 28 de junho de 2019]. Disponível em: https://www.inca.gov.br/noticias/prevencao-e-deteccaoprecoce-cancer-cabeca-e-pescoco-sao-tema-encontro-no-inca

3. Kubrak C, Martin L, Gramlich L, Scrimger R, Jha N, Debenham $\mathrm{B}$, et al. Prevalence and prognostic significance of malnutrition in patients with cancers of the head and neck. Clin Nutr. 2020;39(3):901-9.

4. Gorenc M, Kozjek NR, Strojan P. Malnutrition and cachexia in patients with head and neck cancer treated with (chemo) radiotherapy. Rep Pract Oncol Radiother. 2015;20(4):249-58.

5. Jager-Wittenaar H, Dijkstra PU, Vissink A, van der Laan BF, van Oort RP, Roodenburg JL. Critical weight loss in head and neck cancer: prevalence and risk factors at diagnosis: an explorative study. Support Care Cancer. 2007;15(9):1045-50.

6. Alshadwi A, Nadershah M, Carlson ER, Young LS, Burke PA, Daley BJ. Nutritional considerations for head and neck cancer patients: a review of the literature. J Oral Maxillofac Surg. 2013;71(11):1853-60.

7. Medeiras FPP, Martinez CE, Cardoso SS. Estado nutricional e ingestão alimentar de pacientes com câncer de cabeça e pescoço submetidos a tratamento oncológico. Arq Ciênc Saúde. 2016;23(4):43-7.

8. Alhambra Expósito MR, Herrera-Martínez AD, Manzano García G, Espinosa Calvo M, Bueno Serrano CM, Gálvez Moreno MÁ. Early nutrition support therapy in patients with head-neck cancer. Nutr Hosp. 2018;35(3):505-10.

9. Brasil. Ministério da Saúde. Portal do Governo Brasileiro. IMC em adultos [Internet]. Brasília; 30 de Maio de 2017 [atualizado em 30 de Maio de 2017; citado em 28 de junho de 2019]. Disponível em: http://www.saude.gov.br/component/content/ article/804-imc/40509-imc-em-adultos

10. Organización Panamericana de la Salud. División de Promoción y Protección de la Salud (HPP). Encuesta Multicentrica salud beinestar y envejecimiento (SABE) em América Latina el Caribe: informe preliminar [Internet]. In: XXXVI Reunión del Comité asesor de investigaciones em Salud; 9-11 jun 2001; Kingston, Jamaica: OPAS, 2002 [citado em 28 de junho de 2019]. Disponível em: http://www1.paho.org/Spanish/HDP/ HDR/CAIS-01-05.PDF 
11. Blackburn GL, Bistrian BR, Maini BS, Schlamm HT, Smith MF. Nutritional and metabolic assessment of the hospitalized patient. JPEN J Parenter Enteral Nutr. 1977;1(1):11-22.

12. Mulasi U, Vock DM, Kuchnia AJ, Jha G, Fujioka N, Rudrapatna $\mathrm{V}$, et al. Malnutrition identified by the Academy of Nutrition and Dietetics and American Society for parenteral and enteral nutrition consensus criteria and other bedside tools is highly prevalent in a sample of individuals undergoing treatment for head and neck cancer. JPEN J Parenter Enteral Nutr. 2018;42(1):139-47.

13. Perea LME, Peres MA, Boing AF, Antunes JLF. Tendência de mortalidade por câncer de boca e faringe no Brasil no período 2002-2013. Rev Saude Publica. 2018;52:10.

14. Takara TFM, Morikawa W, Vivacqua RR, Trevisan C, Ando ET, Carvalho GM, et al. Avaliação nutricional em pacientes com câncer de cabeça e pescoço. Rev Bras Cir Cabeça Pescoço. 2012;41(2):70-4.

15. Theme Filha MM, Souza Junior PRB, Damacena GN, Szwarcwald CL. Prevalência de doenças crônicas não transmissíveis e associação com autoavaliação de saúde: Pesquisa Nacional de Saúde, 2013. Rev Bras Epidemiol. [online]. 2015;18(suppl. 2):83-96.
16. Sousa CA, César CLG, Barros MBA, Carandina L, Goldbaum M, Pereira JCR. Doença pulmonar obstrutiva crônica e fatores associados em São Paulo, SP, 2008-2009. Rev Saúde Pública [online]. 2011;45(5):887-96.

17. Alshadwi A, Nadershah M, Carlson ER, Young LS, Burke PA, Daley BJ. Nutritional considerations for head and neck cancer patients: a review of the literature. J Oral Maxillofac Surg. 2013;71(11):1853-60.

18. Kubrak C, Olson K, Jha N, Scrimger R, Parliament M, McCargar L, et al. Clinical determinants of weight loss in patients receiving radiation and chemoirradiation for head and neck cancer: a prospective longitudinal view. Head Neck. 2013;35(5):695-703.

19. Gevaerd SR, Fabre MES, Burigo T, Carneiro CM, Medina LR, Pastore JA, et al. Impacto da terapia nutricional enteral ambulatorial em pacientes oncológicos. Rev Bras Nutr Clin. 2008;23(1):41-5.

20. Camargo S, Oselame GB, Neves EB. Influência da sondagem nasogástrica precoce no índice de massa corporal de portadores de neoplasias de cabeça e pescoço. J Health Sci Inst. 2014;32(1):48-52.

\section{Local de realização do estudo: Universidade Federal de São Paulo - campus São Paulo, São Paulo, SP, Brasil.}

Conflito de interesse: Os autores declaram não haver.

Trabalho apresentado no $23^{\circ}$ Congresso Brasileiro de Nutrição Parenteral e Enteral, realizado em Foz do Iguaçu, PR, Brasil, em 21 de outubro de 2019. 